\title{
Review
}

\section{Pulse Oximetry as a Diagnostic Tool to Determine Pulp Vitality: A Systematic Review}

\author{
Alicia Almudever-Garcia ${ }^{1}{ }^{1}$, Leopoldo Forner ${ }^{1}$, José Luis Sanz ${ }^{1}{ }^{1}$, Carmen Llena ${ }^{1}$, \\ Francisco Javier Rodríguez-Lozano ${ }^{2,3}\left[\mathbb{D}\right.$, Julia Guerrero-Gironés ${ }^{2,3, * \mathbb{C}}$ and María Melo ${ }^{1}$ \\ 1 Departament d'Estomatologia, Facultat de Medicina I Odontologia, Universitat de València, \\ 46010 Valencia, Spain; aag1094@gmail.com (A.A.-G.); leopoldo.forner@uv.es (L.F.); \\ jsanzalex96@gmail.com (J.L.S.); maria.c.llena@uv.es (C.L.); maria.melo.alminana@gmail.com (M.M.) \\ 2 Cellular Therapy and Hematopoietic Transplant Research Group, Biomedical Research Institute, \\ Virgen de la Arrixaca Clinical University Hospital, IMIB-Arrixaca, University of Murcia, \\ 30120 Murcia, Spain; fcojavier@um.es \\ 3 Department of Dermatology, Stomatology, Radiology and Physical Medicine, Morales Meseguer Hospital, \\ Faculty of Medicine, University of Murcia, 30100 Murcia, Spain \\ * Correspondence: julia.guerrero@um.es; Tel.: +34-868-889-158
}

check for updates

Citation: Almudever-Garcia, A.; Forner, L.; Sanz, J.L.; Llena, C.; Rodríguez-Lozano, F.J.; Guerrero-Gironés, J.; Melo, M. Pulse Oximetry as a Diagnostic Tool to Determine Pulp Vitality: A Systematic Review. Appl. Sci. 2021, 11, 2747. https://doi.org/10.3390/ app11062747

Academic Editor: Giovanni Bruno

Received: 19 February 2021

Accepted: 15 March 2021

Published: 18 March 2021

Publisher's Note: MDPI stays neutral with regard to jurisdictional claims in published maps and institutional affiliations.

Copyright: (c) 2021 by the authors. Licensee MDPI, Basel, Switzerland. This article is an open access article distributed under the terms and conditions of the Creative Commons Attribution (CC BY) license (https:/ / creativecommons.org/licenses/by/ $4.0 /)$.
Featured Application: The present systematic review may act as preliminary evidence for the potential application of pulse oximetry as a diagnostic tool for the objective measurement of pulp vitality.

Abstract: Pulse oximetry (PO) can be used as a technique to assess vascularization. Although it is commonly used in the field of medicine, it still has limited use in dentistry. The aim of this study is to evaluate, by means of a systematic review, if pulse oximetry is a viable and effective system to determine pulp vitality and assess the clinical criteria for its use. A literature search was performed in Medline, Scopus, Embase, and Web of Science databases. Clinical studies using pulse oximetry as a tool to determine pulp vitality in permanent teeth were eligible. Quality assessment of the included studies was performed following the Quality Assessment for Diagnostic Accuracy Studies (QUADAS-2) guidelines. A total of 24 articles were included in the review. The subjective test most compared to PO was the cold test. PO has shown a good sensibility and specificity, and it has been used in different clinical situations. Pulse oximetry has a high potential as a tool for the diagnosis of pulp vitality. However, the absence of a specific pulse oximeter for a dental practice, among other limitations, added to the lack of evidence on how different systemic and oral pathologies may affect the pulp's oxygen saturation levels, which hinders its clinical use.

Keywords: diagnosis; endodontics; pulse oximetry; pulp vitality; systematic review

\section{Introduction}

A precise diagnosis of the state of the tooth is an essential procedure previous to any dental treatment. A thorough anamnesis, oral and radiological examination, photographic study, and other complementary tests, such as the evaluation of pain to percussion and palpation, and pulp vitality tests, provide essential information to aid with the diagnostic process [1]. Since performing a histological study on the dental pulp without harming it is impracticable, alternative and non-invasive diagnostic procedures become necessary, such as pulp vitality tests, which can be subjective or objective [2].

Subjective tests (cold, heat, and electric pulp tests (EPTs)) evaluate pulp response through nerve stimulation and are thus often categorized as sensitivity tests. Because these tests rely on the patients' response and the dentists' interpretation of the results, there methods are susceptible to error [3]. Regularly, these tests fail to determine the true vitality of the pulp on primary teeth or after dental trauma, providing false positives or negatives that could lead to an incorrect diagnosis [4]. 
Current objective tests, however, measure the true vitality indicator, namely, pulpal blood flow, and thus may offer higher results in the statistical measures of performance, i.e., sensitivity and specificity. These methods include photoplethysmography, dual-wavelength spectrophotometry, thermography, and, most commonly, laser Doppler flowmetry, pulse oximetry, etc. [5,6].

Pulse oximetry is a non-invasive method that is based on the Beer-Lambert law, which states that "An unknown concentration of solute (hemoglobin) dissolved in a known solvent (blood) can be assessed by the light absorption of the solute" [7] (p. 329). Pulse oximetry systems consist of a sensor with two diodes of different wavelengths that will be absorbed by deoxygenated and oxygenated hemoglobin (red light $(600 \mathrm{~nm})$ and infrared light (940 nm), respectively), and a photoreceptor connected to a microprocessor, which captures the emissions and obtains the pulp oxygen saturation $\left(\mathrm{SpO}_{2}\right)$ and pulse data. Because pulp blood flow is pulsatile, this creates constant changes in the absorption of light by the photoreceptor, which will determine the $\mathrm{SpO}_{2}$ levels comparing the data obtained with the previously established curves of oxygenated and deoxygenated hemoglobin [8].

Currently, no specific pulse oximeter is commercially available for clinical use in the field of dentistry. However, the use of this technique has been investigated as a diagnostic tool for pulp vitality. In these investigations, the absence of a standardized pulse oximeter results in the use and assessment of custom-made probe designs for the pulse oximeter sensors [9-11]. The heterogeneity of these designs added to the methodological differences between studies hinders the applicability of pulse oximetry to the clinical setting. Thus, providing a qualitative synthesis of the available evidence regarding this matter is essential in order to determine the potential use of pulse oximetry as a diagnostic tool.

\section{Materials and Methods}

The present review followed the Preferred Reporting Items for Systematic Reviews and Meta-Analyses (PRISMA) protocol [12] and was registered in the PROSPERO database, under the reference CRD42020157819.

\subsection{Search Strategy}

A systematic electronic search was performed in Medline, Embase, Scopus, and Web of Science databases on 1 June 2020 and last updated on 3 November 2020, without year or language restrictions. The following descriptors were used: "pulse oximetry", "pulse oximeter", "pulp vitality test", "pulp vitality", "vitality", "endod *". Boolean operators "OR" and "AND" were used to combine the search terms. The advanced search strategy for the different databases, along with its findings, are summarized in Table 1.

The literature search, study selection, data extraction, and quality assessment were performed by two independent examiners (A.A. and L.F.). In the event of any disagreement, a third examiner was consulted (J.L.S.).

\subsection{Eligibility Criteria}

Clinical studies assessing the use of pulse oximetry as a tool to determine pulp vitality in permanent teeth were eligible. Both randomized and non-randomized study designs were accepted. Variables regarding patients (i.e., age, gender, medical condition/s, etc.) and teeth (i.e., inflammatory state, tooth type, etc.) were not considered in the selection process. In vivo studies performed in animal models were not included. Non-comparative studies, case reports, and non-clinical studies (i.e., reviews, in vitro assays) were also excluded.

The previously mentioned criteria were established in accordance with the PICOS framework [13] as follows: population/problem (P): patients with permanent teeth; intervention (I): pulse oximetry; comparison/control (C): other diagnostic methods; outcome (O): pulp vitality; study design (S): clinical trials. 
Table 1. Search strategy and findings per database.

\begin{tabular}{clc}
\hline Database & \multicolumn{1}{c}{ Search Strategy } & Findings \\
\hline Medline & \#1 (pulse oximetry OR pulse oximeter) & 20.599 \\
& \#2 (pulp vitality test?) OR (pulp vitality OR vitality OR endod *) & 81.205 \\
Web of Science & \#1 AND \#2 (pulse oximetry OR pulse oximeter) & 83 \\
& \#2 TS = (pulp vitality test? OR pulp vitality OR vitality OR & 10.241 \\
& endod *) & 55.169 \\
Scopus & \#1 AND \#2 & 65 \\
& \#1 ALL (pulse oximetry OR pulse oximeter) & 5.028 \\
& \#2 ALL (pulp vitality test? OR pulp vitality OR vitality OR & 1.305 \\
& endod *) & 89 \\
& \#1 AND \#2 & 22.203 \\
& \#1 (pulse oximetry OR pulse oximeter) & 105.375 \\
& \#2 (pulp vitality test? OR pulp vitality OR vitality OR endod *) & 75 \\
\hline
\end{tabular}

\subsection{Study Selection}

Once the search was performed, the studies were imported to Mendeley reference management software (Elsevier, AMS, Amsterdam, the Netherlands), where duplicates were removed manually. After discarding repeated studies, a preliminary screening of record titles and abstracts was carried out following the aforementioned criteria. Those that fulfilled the criteria were then evaluated for eligibility by full-text screening.

\subsection{Data Extraction}

Each article was analyzed to extract data regarding bibliometric characteristics, methodology, and results. With reference to bibliometric characteristics, the following data were extracted: author, country, institution, year of publication, and journal. With regards to study methodology, extracted variables were pulp vitality tests and measurements assessed, patient variables (number, age, and gender), sample number (teeth), type of teeth, and pulse oximeter name and manufacturer. Regarding study results, pulp vitality results or outcomes were presented as oxygen saturation percentages $\left(\% \mathrm{SpO}_{2}\right)$ and categorized by teeth and by pulp status. A qualitative synthesis of the general outcomes of the included studies was also performed.

\subsection{Quality Assessment}

The risk of bias was performed using the Quality Assessment for Diagnostic Accuracy Studies (QUADAS-2) tool [14]. This guideline is recommended by the Cochrane collaboration for the quality assessment of diagnostic accuracy studies included in a systematic review.

The full texts of the included studies were screened and evaluated for each of the parameters included in the QUADAS-2 checklist. The tool focuses on two aspects-risk of bias (with four domains: patient selection, index test, reference standard, and flow and timing) and applicability concerns (with three domains, namely, patient selection, index test, and reference standard). Each domain is rated as having low or high risk.

\section{Results}

\subsection{Search Results and Study Selection}

The search produced 312 results. After removing duplicates, 151 articles were identified. Of these, 161 were excluded at the title and abstract screening stage. The resulting 25 articles were evaluated by reading their full text, and one article was excluded because it was an in vitro study. A total of 24 articles matched the inclusion criteria and were included in the systematic review (Figure 1). 

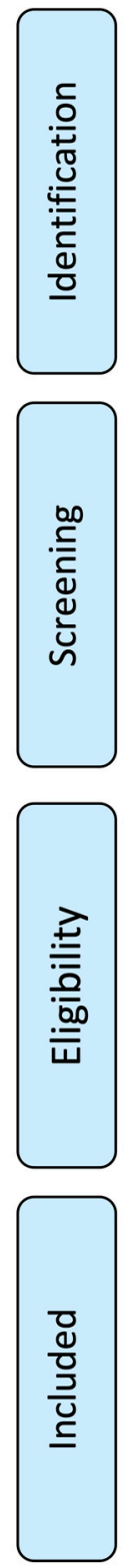

Figure 1. Preferred Reporting Items for Systematic Reviews and Meta-Analyses (PRISMA) flow diagram.

\subsection{Study Characteristics and Methodology}

The bibliometric characteristics and methodological variables extracted from the included studies are presented in Tables 2 and 3, respectively.

The bibliometric analysis revealed that the modal country to study pulse oximetry was Brazil, followed by India. With reference to the year of publication, 2017 was the year with the highest number of publications within this framework.

Regarding the comparison between pulp vitality diagnostic tests, the cold test was the most common subjective method used to compare with pulse oximetry (19 times: [2,4,911,15-28]), followed by the electric pulp test (12 times: [2,9-11,16,20,23-25,29-31]). The least compared was the heat test (three times: $[2,9,26])$.

With reference to the study sample size and characteristics, Souza et al. (2017) [28] reported the largest sample size, using 2543 teeth and 359 participants. The smallest sample size was observed in the study by Kahan et al. (1996) [11], who used only 20 teeth, gathered from 10 participants. The age of the participants ranged from 7 years old to 65 years old. 
Only six studies specified the gender of the participants. From them, women represented the modal gender.

As to the tooth types studied, anterior teeth and, in particular, upper incisors, were the most frequently analyzed tooth type. In relation to the use of pulse oximeters among the included studies, the portable BCI 3301 pediatric pulse oximeter (Smiths Medical PM Inc., Waukesha, WI, USA) was the most commonly assessed.

Table 2. Bibliometric information about the selected studies.

\begin{tabular}{|c|c|c|c|c|}
\hline Author & Country & Institution & Journal & Type of Study \\
\hline J. Schnettler (1991) [25] & USA & $\begin{array}{c}\text { Case Western Reserve } \\
\text { University }\end{array}$ & Journal of Endodontics & Cross-Sectional \\
\hline R. Kahan (1996) [11] & UK & University College Hospital & Journal of Endodontics & Cross-Sectional \\
\hline V. Gopikrishna (2007) A [10] & India & $\begin{array}{l}\text { Meenakshi Ammal Dental } \\
\text { College }\end{array}$ & Journal of Endodontics & Cross-Sectional \\
\hline V. Gopikrishna (2007) B [20] & India & $\begin{array}{l}\text { Meenakshi Ammal Dental } \\
\text { College }\end{array}$ & Journal of Endodontics & Cross-Sectional \\
\hline E. Calil (2008) [17] & Brazil & University of São Paulo & $\begin{array}{c}\text { International Endodontic } \\
\text { Journal }\end{array}$ & Cross-Sectional \\
\hline H. Karayilmaz (2011) [29] & Turkey & $\begin{array}{l}\text { Süleyman Demirel } \\
\text { Universitesi }\end{array}$ & $\begin{array}{c}\text { Journal of Oral } \\
\text { Rehabilitation }\end{array}$ & Cross-Sectional \\
\hline S. Kataoka (2011) [21] & Brazil & University of São Paulo & Journal of Endodontics & Cross-Sectional \\
\hline N. Dastmalchi (2012) [9] & Iran & $\begin{array}{c}\text { Mashland University of } \\
\text { Medical Sciences }\end{array}$ & Journal of Endodontics & Cross-Sectional \\
\hline F. Setzer (2012) [26] & USA & University of Pennsylvania & Journal of Endodontics & Cross-Sectional \\
\hline M. Sadique (2014) [32] & India & $\begin{array}{c}\text { Kunhitharuvai Memorial } \\
\text { Charitable Trust Dental } \\
\text { College }\end{array}$ & $\begin{array}{l}\text { Journal of International } \\
\text { Oral Health }\end{array}$ & Cross-Sectional \\
\hline L. Bergesch (2014) [16] & Brazil & Lutheran University of Brazil & Journal of Endodontics & Cross-Sectional \\
\hline J. Stella (2015) [33] & Brazil & Lutheran University of Brazil & Journal of Endodontics & Cross-Sectional \\
\hline D. Kosturkov (2015) [31] & Bulgaria & Medical University of Sofia & Journal of IMAB & Cross-Sectional \\
\hline C. Caldeira (2016) [4] & Brazil & University of São Paulo & $\begin{array}{l}\text { Journal of Clinical and } \\
\text { Diagnostic Research }\end{array}$ & Cross-Sectional \\
\hline H-J Kong (2016) [24] & Korea & $\begin{array}{l}\text { Chungman National } \\
\text { University Hospital }\end{array}$ & $\begin{array}{l}\text { Acta Odontologica } \\
\text { Scandinavica }\end{array}$ & Cross-Sectional \\
\hline S. Kataoka (2016) [22] & Brazil & University of São Paulo & Journal of Endodontics & Cross-Sectional \\
\hline A. Khademi (2017) [23] & Iran & $\begin{array}{l}\text { Torabinejad Dental Center of } \\
\text { Investigation }\end{array}$ & $\begin{array}{c}\text { The Journal of Craniofacial } \\
\text { Surgery }\end{array}$ & Cross-Sectional \\
\hline B. Anusha (2017) [15] & India & $\begin{array}{c}\text { Narayana Dental College } \\
\text { and Hospital }\end{array}$ & $\begin{array}{l}\text { Journal of Clinical and } \\
\text { Diagnostic Research }\end{array}$ & Cross-Sectional \\
\hline C. Estrela (2017) A [18] & Brazil & Ferederal university of Goiás & Brazilian Dental Journal & Cross-Sectional \\
\hline C. Estrela (2017) B [19] & Brazil & Ferederal University of Goiás & Brazilian Dental Journal & Cross-Sectional \\
\hline S. Souza (2017) [28] & Brazil & $\begin{array}{l}\text { Federal University of } \\
\text { Maranhão }\end{array}$ & Journal of Endodontics & Cross-Sectional \\
\hline D. Kosturkov (2017) [30] & Bulgaria & Medical University of Sofia & Acta Medica Bulgarica & Cross-Sectional \\
\hline C. Solda (2018) [27] & Brazil & School of Dentistry & Brazilian Dental Journal & Cross-Sectional \\
\hline K. Janani (2020) [2] & India & $\begin{array}{c}\text { Saveetha Dental College and } \\
\text { Hospital }\end{array}$ & Brazilian Dental Science & Cross-Sectional \\
\hline
\end{tabular}


Table 3. Data extracted from the selected studies.

\begin{tabular}{|c|c|c|c|c|c|c|c|}
\hline Author & Teeth & Sample & Age & Patients & Test & Measure & Pulse Oximeter \\
\hline $\begin{array}{l}\text { J. Schnettler } \\
\text { (1991) [25] }\end{array}$ & UCI & $\begin{array}{l}\text { Study: } 44 \\
\text { PCG: - } \\
\text { NCG: } 5\end{array}$ & - & 49 & $\begin{array}{l}\mathrm{PO} \\
\mathrm{CT} \\
\mathrm{EPT}\end{array}$ & $\mathrm{SpO}_{2}$ & Novametrix pulse oximeter \\
\hline $\begin{array}{l}\text { R. Kahan } \\
\text { (1996) [11] }\end{array}$ & $\begin{array}{l}\text { UCI } \\
\text { LCI }\end{array}$ & $\begin{array}{c}\text { Study: } 20 \\
\text { PCG: } 10 \text { (finger) } \\
\text { NCG: - }\end{array}$ & - & 10 & $\begin{array}{l}\mathrm{PO} \\
\mathrm{CT} \\
\mathrm{EPT}\end{array}$ & $\mathrm{SpO}_{2}$ & $\begin{array}{l}\text { Ohmeda Biox } 3740 \text { (Ohmeda, } \\
\text { BOC HEalth Care, Louisville, } \\
\text { CO, USA) }\end{array}$ \\
\hline $\begin{array}{l}\text { V. } \\
\text { Gopikrishna } \\
\text { (2007) A [10] }\end{array}$ & UI & $\begin{array}{l}\text { Study: } 17 \\
\text { PCG: } 30 \\
\text { NCG: } 30\end{array}$ & - & 47 & $\begin{array}{l}\mathrm{PO} \\
\mathrm{CT}\end{array}$ & $\mathrm{SpO}_{2}$ & $\begin{array}{l}\text { Nellcor OxiMax } 550 \text { 5th } \\
\text { generation (Tycor Healthcare } \\
\text { Group LP) } \\
\text { Nellcor OxiMaxTM Dura-Y } \\
\text { D-YS Sensor (Tyco Healthcare } \\
\text { Group LP). } \\
\text { A probe was fabricated. }\end{array}$ \\
\hline $\begin{array}{l}\text { V. } \\
\text { Gopikrishna } \\
\text { (2007) B [20] }\end{array}$ & $\begin{array}{l}\mathrm{I} \\
\mathrm{C} \\
\mathrm{P}\end{array}$ & $\begin{array}{l}\text { Study: } 80 \\
\text { PCG: } 80 \\
\text { NCG: - }\end{array}$ & - & 80 & $\begin{array}{l}\mathrm{PO} \\
\mathrm{CT}\end{array}$ & $\mathrm{SpO}_{2}$ & $\begin{array}{l}\text { Nellcor OxiMax 550 5th } \\
\text { generation (Tycor Healthcare } \\
\text { Group LP) } \\
\text { Sensor Nellcor OxiMaxTM } \\
\text { Dura-Y D-YS (Tyco } \\
\text { Healthcare Group LP). } \\
\text { A probe was fabricated }\end{array}$ \\
\hline $\begin{array}{l}\text { E. Calil (2008) } \\
\qquad[17]\end{array}$ & $\begin{array}{l}\text { UCI } \\
\text { UC }\end{array}$ & $\begin{array}{l}\text { Study: } 60 \\
\text { PCG: - } \\
\text { NCG: } 10\end{array}$ & $26-38$ & 17 & $\begin{array}{l}\mathrm{PO} \\
\mathrm{CT}\end{array}$ & $\mathrm{SaO}_{2}$ & $\begin{array}{l}\text { Oxygraph System Partner } \\
\text { Ltd.a., (São Caetano do Sul, } \\
\text { SP, Brazil) } \\
\text { Adapted sensor for ental use } \\
\text { (System Partner Ltd.a). }\end{array}$ \\
\hline $\begin{array}{l}\text { H. } \\
\text { Karayilmaz } \\
\text { (2011) [29] }\end{array}$ & $\begin{array}{l}\text { UCI } \\
\text { ULI }\end{array}$ & $\begin{array}{l}\text { Study: } 59 \\
\text { PCG: } 59 \\
\text { NCG: - }\end{array}$ & $12-18$ & $\begin{array}{l}28 \mathrm{w} \\
23 \mathrm{~m}\end{array}$ & $\begin{array}{l}\text { PO } \\
\text { LDF } \\
\text { EPT }\end{array}$ & $\begin{array}{l}\text { Sensitivity } \\
\text { Specificity }\end{array}$ & $\begin{array}{l}\text { Life Scope I, Multiparameter } \\
\text { Bedside Monitor (Modelo } \\
\text { BSM-2301K (Nihon Kohden } \\
\text { Corp., Tokyo, Japan) } \\
\text { A probe was fabricated }\end{array}$ \\
\hline $\begin{array}{l}\text { S. Kataoka } \\
(2011)[21]\end{array}$ & $\begin{array}{l}\text { UI } \\
\text { UC } \\
\text { LI } \\
\text { LC }\end{array}$ & $\begin{array}{l}\text { Study: } 40 \\
\text { PCG: - } \\
\text { NCG: - }\end{array}$ & $35-55$ & 20 & $\begin{array}{l}\mathrm{PO} \\
\mathrm{CT}\end{array}$ & $\mathrm{SpO}_{2}$ & $\begin{array}{l}\text { Oxygraph pulse oximeter } \\
\text { (System Partner, São Paulo, } \\
\text { Brazil) with tyte Y modificed } \\
\text { sensors }\end{array}$ \\
\hline $\begin{array}{c}\text { N. } \\
\text { Dastmalchi } \\
(2012)[9]\end{array}$ & $\mathrm{P}$ & $\begin{array}{l}\text { Study: } 24 \\
\text { PCG: } 24 \\
\text { NCG: - }\end{array}$ & $18-50$ & 24 & $\begin{array}{l}\text { PO } \\
\text { CT } \\
\mathrm{HT} \\
\mathrm{EPT}\end{array}$ & $\begin{array}{l}\text { Sensitivity } \\
\text { Specificity } \\
\text { PPV } \\
\text { NPV }\end{array}$ & $\begin{array}{l}\text { 520a pulse oximeter, } \\
\text { (Novametrix, Wallingford, CT, } \\
\text { USA) }\end{array}$ \\
\hline $\begin{array}{l}\text { F. Setzer } \\
(2012)[26]\end{array}$ & $\begin{array}{l}\text { UP } \\
\text { LP } \\
\text { UM } \\
\text { LM }\end{array}$ & $\begin{array}{l}\text { Study: } 60 \\
\text { PCG: } 60 \\
\text { NCG: } 60\end{array}$ & $25-55$ & $\begin{array}{l}28 \mathrm{w} \\
30 \mathrm{~m}\end{array}$ & $\begin{array}{l}\mathrm{PO} \\
\mathrm{CT} \\
\mathrm{HT}\end{array}$ & $\mathrm{SpO}_{2}$ & $\begin{array}{l}\text { Oxygraph, System Partner } \\
\text { (São Paulo, Brazil) } \\
\text { Modified type Y sensors }\end{array}$ \\
\hline $\begin{array}{l}\text { M. Sadique } \\
(2014)[32]\end{array}$ & $\begin{array}{l}\text { UCI } \\
\text { ULI } \\
\text { UC }\end{array}$ & $\begin{array}{l}\text { Study: } 60 \\
\text { PCG: } 60 \text { (finger) } \\
\text { NCG: } 30\end{array}$ & $15-40$ & 60 & $\mathrm{PO}$ & $\mathrm{SpO}_{2}$ & $\begin{array}{l}\text { Criticare 504-US oxygen } \\
\text { sensor }\end{array}$ \\
\hline
\end{tabular}


Table 3. Cont

\begin{tabular}{|c|c|c|c|c|c|c|c|}
\hline Author & Teeth & Sample & Age & Patients & Test & Measure & Pulse Oximeter \\
\hline $\begin{array}{l}\text { L. Bergesch } \\
(2014)[16]\end{array}$ & Anterior & $\begin{array}{l}\text { Study: } 67 \\
\text { PCG: } 30 \\
\text { NCG: - }\end{array}$ & $32-64$ & 35 & $\begin{array}{l}\mathrm{PO} \\
\mathrm{CT} \\
\mathrm{EPT}\end{array}$ & $\mathrm{SpO}_{2}$ & $\begin{array}{l}\text { Portabel pediatric pulse } \\
\text { oximeter (3301; Smiths } \\
\text { Medical PM INC., Waukesha, } \\
\text { WI, USA) } \\
\text { Sensors: } 3025 \text { (tooth) y } 3026 \\
\text { (finger) } \\
\text { A probe was fabricated }\end{array}$ \\
\hline $\begin{array}{l}\text { J. Stella (2015) } \\
\text { [33] }\end{array}$ & $\mathrm{CI}$ & $\begin{array}{l}\text { Study: } 110 \\
\text { PCG: - } \\
\text { NCG: - }\end{array}$ & $7-36$ & 57 & $\mathrm{PO}$ & $\mathrm{SpO}_{2}$ & $\begin{array}{l}\text { BCI } 3301 \text { pediatric (Smiths } \\
\text { Medical PM Inc., Waukesha, } \\
\text { WI, USA)Sensor } 3025 \\
\text { A probe was fabricated }\end{array}$ \\
\hline $\begin{array}{l}\text { D. Kosturkov } \\
\text { (2015) [31] }\end{array}$ & Anterior & $\begin{array}{l}\text { Study: } 1058 \\
\text { PCG: - } \\
\text { NCG: - }\end{array}$ & $18-25$ & 31 & $\begin{array}{l}\mathrm{PO} \\
\mathrm{EPT}\end{array}$ & $\mathrm{SpO}_{2}$ & $\begin{array}{l}\text { Pulse oximeter } \\
\text { Conect }^{\mathrm{TM}} \text { _CMS60DSpecial } \\
\text { holder fabricated }\end{array}$ \\
\hline $\begin{array}{l}\text { C. Caldeira } \\
(2016)[4]\end{array}$ & $\begin{array}{l}\mathrm{I} \\
\mathrm{C} \\
\mathrm{P}\end{array}$ & $\begin{array}{l}\text { Study: } 59 \\
\text { PCG: } 46 \\
\text { NCG: } 10\end{array}$ & $14-42$ & 46 & $\begin{array}{l}\mathrm{PO} \\
\mathrm{CT}\end{array}$ & $\mathrm{SpO}_{2}$ & $\begin{array}{l}\text { Oxigraph, System Partner } \\
\text { (Sao Caetano do Sul, Brazil) }\end{array}$ \\
\hline $\begin{array}{l}\text { H-J Kong } \\
(2016)[24]\end{array}$ & UCI & $\begin{array}{l}\text { Study: } 30 \\
\text { PCG: - } \\
\text { NCG: - }\end{array}$ & $24-40$ & 15 & $\begin{array}{l}\mathrm{PO} \\
\mathrm{CT} \\
\mathrm{EPT}\end{array}$ & $\begin{array}{l}\text { Perfusion } \\
\text { index } \\
\mathrm{SpO}_{2}\end{array}$ & $\begin{array}{l}\text { Modified Nellcor sensor } \\
\text { compatible with a comercial } \\
\text { pulse oximeter (MP-570T, } \\
\text { MEKICS Co., Paju, Korea) }\end{array}$ \\
\hline $\begin{array}{l}\text { S. Kataoka } \\
(2016)[22]\end{array}$ & $\mathrm{I}$ & $\begin{array}{l}\text { Study: } 693 \\
\text { PCG: } 693 \\
\text { NCG: - }\end{array}$ & $35-65$ & 180 & $\begin{array}{l}\mathrm{PO} \\
\mathrm{CT}\end{array}$ & $\mathrm{SpO}_{2}$ & $\begin{array}{l}\text { Oxygraph pulse oximeter } \\
\text { (System Partner, São Paulo, } \\
\text { Brazil) with type Y modified } \\
\text { sensors. }\end{array}$ \\
\hline $\begin{array}{l}\text { A. Khademi } \\
\text { (2017) [23] }\end{array}$ & UC & $\begin{array}{l}\text { PCG: } 20 \\
\text { NCG: } 20\end{array}$ & $13-24$ & $\begin{array}{l}11 \mathrm{w} \\
9 \mathrm{~m}\end{array}$ & $\begin{array}{l}\mathrm{PO} \\
\mathrm{CT} \\
\mathrm{EPT}\end{array}$ & $\mathrm{SpO}_{2}$ & $\begin{array}{l}\text { Criticare } 504 \text { (Criticare, } \\
\text { Waukesha, WI) }\end{array}$ \\
\hline $\begin{array}{l}\text { B. Anusha } \\
(2017)[15]\end{array}$ & $\begin{array}{l}\text { UCI } \\
\text { ULI } \\
\text { UC } \\
\text { LCI } \\
\text { LLI } \\
\text { LC }\end{array}$ & $\begin{array}{l}\text { Study: } 60 \\
\text { PCG: } 20 \\
\text { NCG: } 20\end{array}$ & $25-40$ & $\begin{array}{l}48 w \\
52 \mathrm{~m}\end{array}$ & $\begin{array}{l}\mathrm{PO} \\
\mathrm{CT} \\
\mathrm{GT}\end{array}$ & $\mathrm{SpO}_{2}$ & $\begin{array}{l}\text { Monitor: B20, GE, Finland } \\
\text { Sensor: B20, GE, Finland }\end{array}$ \\
\hline $\begin{array}{l}\text { C. Estrela } \\
\text { (2017) A [18] }\end{array}$ & $\begin{array}{l}\text { UM } \\
\text { LM }\end{array}$ & $\begin{array}{l}\text { Study: } 112 \\
\text { PCG: - } \\
\text { NCG: } 10\end{array}$ & $17-40$ & $\begin{array}{l}11 \mathrm{w} \\
11 \mathrm{~m}\end{array}$ & $\begin{array}{l}\mathrm{PO} \\
\mathrm{CT}\end{array}$ & $\mathrm{SaO}_{2}$ & $\begin{array}{l}\text { BCI } 3301 \text { pediatric (Smiths } \\
\text { Medical PM Inc., Waukesha, } \\
\text { WI, USA) } \\
\text { Sensor: } 3025 \text { (tooth), } 3026 \\
\text { (finger) } \\
\text { A probe was fabricated }\end{array}$ \\
\hline $\begin{array}{c}\text { C. Estrela } \\
\text { (2017) B [19] }\end{array}$ & UP & $\begin{array}{l}\text { Study: } 120 \\
\text { PCG: - } \\
\text { NCG: - }\end{array}$ & $20-44$ & 100 & $\begin{array}{l}\mathrm{PO} \\
\mathrm{CT}\end{array}$ & $\mathrm{SaO}_{2}$ & $\begin{array}{l}\text { BCI } 3301 \text { pediatric (Smiths } \\
\text { Medical PM Inc., Waukesha, } \\
\text { WI, USA) } \\
\text { Sensor: } 3025 \text { (tooth), } 3026 \\
\text { (finger) } \\
\text { A probe was fabricated }\end{array}$ \\
\hline $\begin{array}{l}\text { S. Souza } \\
(2017)[28]\end{array}$ & $\begin{array}{l}\mathrm{I} \\
\mathrm{C} \\
\mathrm{P} \\
\mathrm{M}\end{array}$ & $\begin{array}{l}\text { Study: } 728 \\
\text { PCG: - } \\
\text { NCG: } 1815\end{array}$ & $\begin{array}{l}\text { Mean: } \\
30\end{array}$ & 359 & $\begin{array}{l}\mathrm{PO} \\
\mathrm{CT}\end{array}$ & $\mathrm{SpO}_{2}$ & $\begin{array}{l}\text { MD300A (IMFtec Tecnologia } \\
\text { para Saúde LTDA, São Paulo, } \\
\text { SP, Brazil) }\end{array}$ \\
\hline $\begin{array}{l}\text { D. Kosturkov } \\
\text { (2017) [30] }\end{array}$ & $\begin{array}{l}\text { Anterior } \\
\text { and } \\
\text { posterior }\end{array}$ & $\begin{array}{l}\text { Study: } 31 \\
\text { PCG: } 78 \\
\text { NCG: - }\end{array}$ & - & 45 & $\begin{array}{l}\mathrm{PO} \\
\mathrm{EPT}\end{array}$ & $\mathrm{SpO}_{2}$ & $\begin{array}{l}\text { Contect CMS 60D with } \\
\text { modified probe }\end{array}$ \\
\hline
\end{tabular}


Table 3. Cont.

\begin{tabular}{|c|c|c|c|c|c|c|c|}
\hline Author & Teeth & Sample & Age & Patients & Test & Measure & Pulse Oximeter \\
\hline $\begin{array}{l}\text { C. Solda } \\
\text { (2018) [27] }\end{array}$ & UCI & $\begin{array}{c}\text { Study: } 68 \\
\text { PCG: - } \\
\text { NCG: } 10\end{array}$ & $19-36$ & $\begin{array}{l}41 \mathrm{w} \\
27 \mathrm{~m}\end{array}$ & $\begin{array}{l}\mathrm{PO} \\
\mathrm{CT}\end{array}$ & $\mathrm{SaO}_{2}$ & $\begin{array}{l}\text { Portable pediatric pulse } \\
\text { oximeter (BCI 3301, Smiths } \\
\text { Medical PM Inc., Waukesha, } \\
\text { WI, USA) } \\
3026 \text { sensors (for fingers) } \\
\text { Adapter specifically } \\
\text { fabricated }\end{array}$ \\
\hline $\begin{array}{l}\text { K. Janani } \\
(2020) \text { [2] }\end{array}$ & $\begin{array}{c}\mathrm{I} \\
\mathrm{C} \\
\mathrm{LP}\end{array}$ & $\begin{array}{c}\text { Study: } 37 \\
\text { PCN: Finger (17) } \\
\text { NCG: - }\end{array}$ & $18-50$ & 37 & $\begin{array}{l}\mathrm{PO} \\
\mathrm{CT} \\
\mathrm{HT} \\
\mathrm{EPT}\end{array}$ & $\mathrm{SpO}_{2}$ & $\begin{array}{l}\text { Monitor: Nellcor N-600 } \\
\text { (Healthcare group LP, } \\
\text { Pleasanton, CA, USA). } \\
\text { Sensor: N3 oxy sensor } \\
\text { (Healthcare group LP, } \\
\text { Pleasanton, CA, USA). } \\
\text { Sensor holder designed and } \\
\text { custom made. }\end{array}$ \\
\hline
\end{tabular}

I: incisors; C: canines; P: premolars; M: molars; UCI: upper central incisors; ULI: upper lateral incisors; UC: upper canines; LCI: lower central incisors; LLI: lower lateral incisors; UI: upper incisors; LI: lower incisors; LC: lower canines; UP: upper premolars; LP: lower premolars; UM: upper molars; LM: lower molars; PO: pulse oximetry; CT: cold test; HT: heat test; EPT: electric pulp test; LDF: laser Doppler flowmetry; PCN: positive control group; NCG: negative control group; $\mathrm{SpO}_{2}$ : pulp oxygen saturation level; PPV: positive predictive value; NPV: negative predictive value; w: woman; m: men.

\subsection{Study Results}

All authors expressed their results as percentages of oxygen saturation levels, except for Khademi et al. (2017) [23], who measured the pulpal oxygen saturation of maxillary canines after alveolar cleft bone grafting in $\mathrm{mmHg}$, using the healthy contralateral canine as the control group. They reported $85.04 \mathrm{mmHg}$ in the affected canine, and $87.78 \mathrm{mmHg}$ in the healthy canine when using a Criticare 504 pulse oximeter (Criticare, Waukesha, WI, USA).

A total of eight studies reported pulp vitality test outcomes as $\mathrm{SpO}_{2}$ levels by type of tooth $[11,17,18,27,28,31-33]$. Moreover, $\% \mathrm{SpO}_{2}$ values reported by the previously mentioned studies, categorized by tooth type, along with their sample numbers, are presented in Table 4. All studies found PO reliable regardless of the tooth type, although studies that compared maxillary and mandibular teeth generally found higher levels of $\mathrm{SpO}_{2}$ in mandibular teeth.

Pulse oximetry was also assessed as a tool for the diagnosis of pulp vitality at different stages of pulp inflammation, i.e., reversible pulpitis, irreversible pulpitis, necrotic pulp. Setzer et al. (2012) [26] and Anusha et al. (2017) [15] compared PO to thermal tests; Caldeira et al. (2016) [4] compared it only to the cold test, and Kosturkov and Uzunov (2017) [30] compared PO to the EPT. In all cases, the studies measured $\mathrm{SpO}_{2}$ in different pulpar inflammatory states, producing statistically significant results and consequently concluding that PO was a valid diagnostic tool. These results, categorized by pulp status, are summarized in Table 5.

The same was observed in studies evaluating the effectiveness of PO to determine pulp vitality after trauma $[2,4,10]$. In all of the studies, after assessing the changes in $\mathrm{SPO}_{2}$ in traumatized teeth at different time periods, it was concluded that PO was a viable diagnostic tool in these cases.

Two studies investigated the effect of head and neck radiotherapy (RT) on $\mathrm{SPO}_{2}$ using pulse oximetry [21,22]. While Kataoka et al. (2016) [22] studied the levels of $\% \mathrm{opO}_{2}$ by type of teeth, in the study of 2011 [21], they calculated the $\mathrm{SpO}_{2}$ levels at different stages of radiotherapy (RT). Therefore, at the first time point (before the RT), the mean percentage of $\mathrm{SpO}_{2}$ of the assessed teeth was 93\%, at the second time point (beginning of RT with radiation doses between $30 \mathrm{~Gy}$ and $35 \mathrm{~Gy}$ ) $83 \%$, at the third time point (end of RT with radiation doses between $60 \mathrm{~Gy}$ and $70 \mathrm{~Gy}$ ) $77 \%$, and at the fourth time point (four months 
to five months after the beginning of cancer treatment) $84 \%$. Both studies concluded that the pulp maintained its regeneration potential after RT.

Table 4. Oxygen saturation percentages $\left(\% \mathrm{SpO}_{2}\right)$ by tooth type.

\begin{tabular}{|c|c|c|c|c|c|c|c|c|}
\hline Author & UI & UC & UP & UM & LI & LC & LP & $\mathbf{L M}$ \\
\hline $\begin{array}{l}\text { R. Kahan } \\
\text { (1996) [11] }\end{array}$ & $28.95 \%(10)$ & - & - & - & $50.28 \%(10)$ & - & - & - \\
\hline $\begin{array}{c}\text { E. Calil } \\
(2008)[17]\end{array}$ & $91.29 \%(30)$ & $90.69 \%(30)$ & - & - & - & - & - & - \\
\hline $\begin{array}{l}\text { M. Sadique } \\
\text { (2014) [32] }\end{array}$ & $\begin{array}{c}\text { UCI: } \\
85.11 \%(15) \\
\text { ULI: } 80.21 \% \\
(15)\end{array}$ & $89.55 \%$ & - & - & - & - & - & - \\
\hline $\begin{array}{c}\text { J. Stella } \\
(2015)[33]\end{array}$ & $81.25 \%$ & - & - & - & - & - & - & - \\
\hline $\begin{array}{c}\text { D. } \\
\text { Kosturkov } \\
(2015)[31]\end{array}$ & $\begin{array}{l}\text { UCI: } 84.4 \% \\
\text { ULI: } 83.4 \%\end{array}$ & $83.4 \%$ & - & - & $\begin{array}{c}\text { LCI: } 83.5 \% \\
\text { LLI: } 82 \%\end{array}$ & $84.5 \%$ & - & - \\
\hline $\begin{array}{l}\text { S. Kataoka } \\
\text { (2016) [22] }\end{array}$ & $\begin{array}{c}\text { SG } \\
\text { UCI: } \\
\text { 89-98\% } \\
\text { ULI: } \\
90-98 \% \\
\text { CG } \\
\text { UCI: } \\
89-98 \% \\
\text { ULI: } \\
\text { 90-98\% }\end{array}$ & $\begin{array}{c}\text { SG: } 89-96 \% \\
\text { CG: } \\
\text { 89-95\% }\end{array}$ & - & - & $\begin{array}{c}\text { SG } \\
\text { LCI: } \\
\text { 89-96\% } \\
\text { LLI: } \\
88-96 \% \\
\text { CG } \\
\text { LCI: } \\
\text { 89-96\% } \\
\text { LLI: } \\
\text { 89-97\% }\end{array}$ & $\begin{array}{c}\text { SG: } 89-95 \% \\
\text { CG: } \\
90-96 \%\end{array}$ & - & - \\
\hline $\begin{array}{c}\text { C. Estrela } \\
\text { (2017) A } \\
\text { [18] }\end{array}$ & - & - & - & $83.59 \%(61)$ & - & - & - & $86.89 \%$ (51) \\
\hline $\begin{array}{l}\text { S. Souza } \\
(2017)[28]\end{array}$ & $\begin{array}{c}\text { HbSS } \\
\text { UCI: 78\% } \\
\text { ULI: 79\% } \\
\text { HbAA } \\
\text { UCI: 89\% } \\
\text { ULI: } 87 \%\end{array}$ & $\begin{array}{c}\text { HbSS: } 87 \% \\
\text { HbAA: } \\
86 \%\end{array}$ & $\begin{array}{c}\text { HbSS: } 87 \% \\
\text { HbAA: } \\
91 \%\end{array}$ & $\begin{array}{c}\text { HbSS: } 75 \% \\
\text { HbAA: } \\
89 \%\end{array}$ & $\begin{array}{c}\text { HbSS } \\
\text { LCI: } 86 \% \\
\text { LLI: } 93 \% \\
\text { HbAA: } \\
\text { LCI:83\% } \\
\text { LLI:91\% }\end{array}$ & $\begin{array}{c}\text { HbSS: } 93 \% \\
\text { HbAA: } \\
90 \%\end{array}$ & $\begin{array}{c}\text { HbSS: } 88 \% \\
\text { HbAA: } \\
88 \%\end{array}$ & $\begin{array}{c}\text { HbSS: } 89 \% \\
\text { HbAA: } \\
89 \%\end{array}$ \\
\hline
\end{tabular}

\section{Solda UCI: $84.9 \%$}

(2018) [27] ULI: $85.4 \%$

UI: upper incisors; UCI: upper central incisors; ULI: upper lateral incisors; UC: upper canines; UP: upper premolars; UM: upper molars; LI: lower incisors; LCI: lower central incisors; LLI: lower lateral incisors; LC: lower canines; LP: lower premolars; LM: lower molars; SC: study group; CG: control group; HbSS: individuals with sickle cell anemia; HbAA: individuals with normal hemoglobin A.

Table 5. Oxygen saturation percentages $\left(\% \mathrm{SpO}_{2}\right)$ by pulp status.

\begin{tabular}{cccccc}
\hline Author & PC & RP & IP & PN & NC \\
\hline $\begin{array}{c}\text { F. Setzer } \\
\text { (2012) [26] }\end{array}$ & $92.2 \%$ & $87.4 \%$ & $83.1 \%$ & $74.6 \%$ & $0 \%$ \\
$\begin{array}{c}\text { C. Caldeira } \\
\quad \text { (2016) [4] }\end{array}$ & $93 \%$ & $92 \%$ & $89 \%$ & $71 \%$ & $0 \%$ \\
$\begin{array}{c}\text { B. Anusha } \\
\begin{array}{c}\text { (2017) [15] } \\
\text { D. Kosturkov }\end{array}\end{array}$ & $94.6 \%$ & $85.45 \%$ & $81.6 \%$ & $70.7 \%$ & $0 \%$ \\
$\quad 81.47 \%$ & $93.81 \%$ & - & - & - \\
\hline
\end{tabular}


Various studies assessed the use of pulse oximetry to determine pulp vitality in patients with different pathologies. Bergesch et al. (2014) [16] studied how the periodontal disease affected $\mathrm{SpO}_{2}$ levels and found out that patients with periodontal disease had fewer levels of $\mathrm{SpO}_{2} . \mathrm{SpO}_{2}$ in periodontal patients was $76.7 \%$, whereas in healthy patients it was significantly higher (86.7\%). In a similar manner, Souza et al. (2017) [28] investigated $\mathrm{SpO}_{2}$ on patients with homozygous sickle cell anemia, and Khademi et al. (2017) [23] compared PO to EPT and cold tests in canines after alveolar cleft bone grafting. In the first study, they concluded that patients with sickle cell anemia had lower levels of $\mathrm{SpO}_{2}$ than those without it, except in canines. In the second study, even though both sides had adequate blood flow supply, $\mathrm{SpO}_{2}$ levels were lower on the cleft side.

Singularly, Solda et al. (2018) [27] studied the levels of $\mathrm{SaO}_{2}$ in maxillary central incisors that underwent an at-home bleaching treatment with $10 \%$ carbamide peroxide used daily for $4 \mathrm{~h}$, concluding that, even though at first it caused a decrease in $\mathrm{SaO}_{2}$ levels, it returned to normal levels 30 days after the treatment was finished.

Gopikrishna et al. (2007b) [20], Karayilmaz and Kirzioglu (2011) [29], Dastmalchi et al. (2012) [9], and Janani et al. (2020) [2] studied the sensitivity, specificity, positive predictive value (PPV), and negative predictive value (NPV) of PO as opposed to other diagnostic methods. Gopikrishna et al. (2007b) [20] concluded that PO had the most sensitivity, specificity, and was the most accurate, and had a PPV of 0.95 and an NPV of 1. For Karayilmaz and Kirzioglu (2011) [29] the test that had the most sensitivity and specificity was also PO (0.813 and 0.949 , respectively), showing a PPV of 0.941 and NPV of 0.835. Dastmalchi et al. (2012) [9] concluded that PO had the most sensitivity (0.93) and the least specificity (1) and that the cold test had the least sensitivity (0.53) and the most specificity (0.66). In this case, the PPV and NPV for PO were 1 and 0.90, respectively. Lastly, Janani et al. (2020) [2] reported that PO had a sensitivity, specificity, PPV, and NPV of 100, being the most accurate of all tests, followed by the cold test, which had a sensitivity of 50 , a specificity of 81.82 , a PPV of 50, and an NPV of 81.82 .

Lastly, Stella et al. (2015) [33] and Estrela et al. (2017b) [19] compared the difference between $\mathrm{SpO}_{2}$ levels by age groups. Stella et al. (2015) [33] only stated the difference between "Adolescents" (84.35\%) and "Adults" (77.88\%). However, Estrela et al. (2017b) [19] made four groups of ages: 20-24 years old (89.71\%), 25-29 years old (87.67\%), 30-34 years old $(88.71 \%)$, 35-39 years old $(84.80 \%)$, and $40-44$ years old $(80 \%)$, and the mean $\mathrm{SpO}_{2}$ was $86.2 \%$.

\subsection{Quality Assessment}

The results of the quality assessment (risk of bias) using the quality assessment for diagnostic accuracy studies tool QUADAS-2 [14] are presented in Table 6. Only four studies blinded their operators $[2,10,20,28]$, and only one study selected their studies randomly [23]. Many studies showed high concerns regarding index test or reference standard, and only four studies showed low risk in both domains [10,16,20,25]. All studies showed low concerns in terms of flow and timing. Regarding applicability concerns, all studies showed a low risk.

Table 6. Quality Assessment for Diagnostic Accuracy Studies (QUADAS)-2 statement checklist.

\begin{tabular}{|c|c|c|c|c|c|c|c|}
\hline \multirow[b]{2}{*}{ Study } & \multicolumn{4}{|c|}{ Risk of Bias } & \multicolumn{3}{|c|}{ Applicability Concerns } \\
\hline & $\begin{array}{l}\text { Patient } \\
\text { Selection }\end{array}$ & Index Test & $\begin{array}{l}\text { Reference } \\
\text { Standard }\end{array}$ & $\begin{array}{c}\text { Flow and } \\
\text { Timing }\end{array}$ & $\begin{array}{c}\text { Patient } \\
\text { Selection }\end{array}$ & Index Test & $\begin{array}{c}\text { Reference } \\
\text { Standard }\end{array}$ \\
\hline J. Schnettler (1991) [25] & (;) & $\overline{(*)}$ & (;) & (;) & (:) & $\overline{(i)}$ & $\overline{(})$ \\
\hline R. Kahan (1996) [11] & 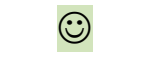 & 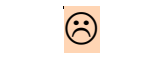 & 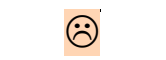 & $\bar{\ominus}$ & (;) & (;) & (;) \\
\hline V. Gopikrishna (2007) A [10] & (;) & (;) & (;) & (;) & (;) & (;) & ;) \\
\hline V. Gopikrishna (2007) B [20] & ;: & (;) & ;: & ;:) & (;) & ;: & $\overline{(;)}$ \\
\hline
\end{tabular}


Table 6. Cont.

\begin{tabular}{|c|c|c|c|c|c|c|c|}
\hline \multirow[b]{2}{*}{ Study } & \multicolumn{4}{|c|}{ Risk of Bias } & \multicolumn{3}{|c|}{ Applicability Concerns } \\
\hline & $\begin{array}{c}\text { Patient } \\
\text { Selection }\end{array}$ & Index Test & $\begin{array}{c}\text { Reference } \\
\text { Standard }\end{array}$ & $\begin{array}{c}\text { Flow and } \\
\text { Timing }\end{array}$ & $\begin{array}{c}\text { Patient } \\
\text { Selection }\end{array}$ & Index Test & $\begin{array}{l}\text { Reference } \\
\text { Standard }\end{array}$ \\
\hline E. Calil (2008) [17] & ;) & ;: & ;) & ;) & ;) & ;) & ;) \\
\hline H. Karayilmaz (2011) [29] & ;) & ;: & 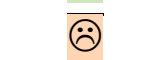 & (:) & ;: & $\bar{\ominus}$ & $\bar{\ominus}$ \\
\hline S. Kataoka (2011) [21] & $\bar{\ominus}$ & 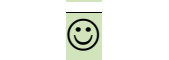 & $\bar{\theta}$ & $\bar{\odot}$ & $\bar{\odot}$ & $\bar{\odot}$ & $\overline{(;)}$ \\
\hline N. Dastmalchi (2012) [9] & (:) & (:) & (:) & $\bar{\ominus}$ & $\bar{\ominus}$ & $\bar{\ominus}$ & $\bar{\ominus}$ \\
\hline F. Setzer (2012) [26] & (:) & $\bar{\ominus}$ & : & $\bar{\ominus}$ & (:) & (:) & (:) \\
\hline M. Sadique (2014) [32] & $\bar{\ominus}$ & ஜ & : & (:) & (:) & $\bar{\ominus}$ & (:) \\
\hline L. Bergesch (2014) [16] & ;: & (:) & $\bar{\ominus}$ & (:) & (:) & (:) & ;) \\
\hline J. Stella (2015) [33] & (;) & $\bar{\ominus}$ & : & (:) & $\overline{0}$ & ;: & (;) \\
\hline D. Kosturkov (2015) [31] & (;) & $\bar{\theta}$ & (:) & $\bar{\ominus}$ & (:) & (:) & (;) \\
\hline C. Caldeira (2016) [4] & $\overline{0}$ & 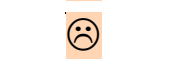 & (:) & (:) & ;) & (;) & (;) \\
\hline H-J Kong (2016) [24] & ;: & $\bar{\ominus}$ & 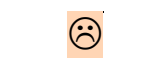 & $\bar{\ominus}$ & $\bar{\ominus}$ & (:) & (;) \\
\hline S. Kataoka (2016) [22] & (:) & $\bar{\ominus}$ & : & $\bar{\ominus}$ & $\bar{\ominus}$ & (:) & $\bar{\theta}$ \\
\hline A. Khademi (2017) [23] & (;) & (:) & (:) & (:) & (:) & (:) & (;) \\
\hline B. Anusha (2017) [15] & (;) & (:) & $\bar{\ominus}$ & $\bar{\ominus}$ & (:) & $\bar{\ominus}$ & (;) \\
\hline C. Estrela (2017) A [18] & (;) & $\bar{\theta}$ & $\bar{\ominus}$ & $\bar{\ominus}$ & $\overline{0}$ & $\overline{(;)}$ & (;) \\
\hline C. Estrela (2017) B [19] & (;) & $\ddot{\theta}$ & (;) & (;) & $\bar{\ominus}$ & $\overline{(;)}$ & $\overline{(i)}$ \\
\hline S. Souza (2017) [28] & (;) & $\bar{\ominus}$ & 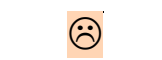 & $\bar{\ominus}$ & $\bar{\ominus}$ & (:) & $\bar{\ominus}$ \\
\hline D. Kosturkov (2017) [30] & (;) & ;: & 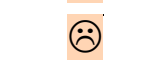 & $\bar{\ominus}$ & ;: & ;) & (:) \\
\hline C. Solda (2018) [27] & ;) & : & $\overline{;}$ & $\bar{\ominus}$ & (:) & $\overline{-i}$ & (;) \\
\hline K. Janani (2020) [2] & $\overline{-i}$ & (:) & $\overline{(;)}$ & $\bar{\odot}$ & ;: & ;) & $\bar{\ominus}$ \\
\hline
\end{tabular}

\section{Discussion}

Generally, pulp vitality tests are sensitivity tests that rely on the patients' response and the dentists' interpretation, which can result in false-positive and negative responses and, thereby, in an incorrect diagnosis that may lead to an inadequate or unnecessary treatment. Pulse oximetry is a non-invasive, objective method that could be potentially used as a diagnostic tool in everyday dental practice. Unlike subjective tests, PO does not stimulate nerve fibers but measures pulpal blood flow, thereby resulting in a more reliable diagnosis. Since it does not generate any pain during the process (unlike heat or cold tests), it can be better tolerated by patients. The use of this technique is yet to be implemented in the clinical setting but has been recently investigated as a potential diagnostic tool. Thus, the aim of the present systematic review was to perform a qualitative synthesis of available studies on the use of pulse oximetry to determine pulp vitality. To the authors' knowledge, this is the first systematic review to assess the potential application of pulse oximetry as a tool for the determination of pulp vitality in dentistry.

All studies used vital teeth to test the efficacy of pulse oximetry, but only Schnettler and Wallace (1991) [25], Gopikrishna et al. (2007a) [10], Calil et al. (2008) [17], Karayilmaz and Kirzioglu (2011) [29], Setzer et al. (2012) [26], Sadique et al. (2014) [32], Caldeira et al. (2016) [4], Anusha et al. (2017) [15], Estrela et al. (2017a) [18], Estrela et al. (2017b) [19], Solda et al. (2018) [27] and Janani et al. (2020) [2] used endodontically treated teeth as negative controls. Kahan et al. (1996) [11], Gopikrishna et al. (2007b) [20], Dastmalchi et al. 
(2012) [9], and Stella et al. (2015) [33] did not have a negative control group; Bergesch et al. (2014) [16] compared healthy teeth to those that had the periodontal disease; Kataoka et al. (2016) [22] studied patients that had undergone radiotherapy treatment and those who had not; Khademi et al. (2017) [23] investigated maxillary canines after alveolar cleft bone grafting, comparing it to the healthy contralateral; Souza et al. (2017) [28] focused on patients with sickle cell anemia and normal hemoglobin A; and Kosturkov and Uzunov (2017) [30] compared intact teeth and teeth with hyperemia pulpae. As for the positive control group, all studies used healthy teeth, except Kahan et al. (1996) [11], Sadique et al. (2014) [32], and Janani et al. (2020) [2], which used the finger as the control group.

Calil et al. (2008) [17], Karayilmaz and Kirzioglu (2011) [29], Stella et al. (2015) [33], Caldeira et al. (2016) [4], Estrela et al. (2017a) [18], Souza et al. (2017) [28], and Solda et al. (2018) [27] calculated the $\mathrm{SpO}_{2}$ correlation of the finger with the teeth; only Caldeira et al. (2016) [4] found a correlation between systemic levels of $\mathrm{SpO}_{2}$ and those of the traumatized teeth.

Pulse oximetry has demonstrated to be a helpful tool in diagnosing pulp vitality in normal circumstances and in patients that had undergone radiotherapy [21,22] with sickle cell anemia [28] after alveolar cleft bone grafting [23], to diagnose different states of pulp inflammation $[4,15,20,26]$ in patients with periodontal disease [16] after an at-home bleaching treatment [27], and in recently traumatized teeth [4,10].

As mentioned previously, four articles studied the sensitivity, specificity, positive predictive value (PPV), and negative predictive value (NPV) of PO as opposed to other diagnostic methods $[2,9,20,29]$, and in all of them, PO showed higher results. A clinical test's sensitivity refers to its ability to identify correctly the patients that have the disease, while specificity refers to the capability of a clinical test to properly identify the patients who do not have the disease. The PPV of a clinical test stands for the probability that a patient with a disease is a true positive, while the NPV is the probability that a patient with a negative result truly is healthy [34]. The aforementioned results act as evidence for the reliability of pulse oximetry as a tool for classifying truly vital and non-vital teeth.

However, pulse oximetry still has many limitations. To obtain a valid result, both diodes need to be parallel, which becomes a challenge because currently there are no pulse oximeters specifically designed for dentistry, and any minor head movement from the patient (such as swallowing) can destabilize the diodes and alter the results [35]. Kahan et al. (1996) [11], Gopikrishna et al. (2007b) [20], and Dastmalchi et al. (2012) [9] fabricated their own probes, in which sensors could be held parallel and fit onto the teeth anatomy. Except for Kahan et al. (1996) [11], who did not find any diagnostic use for pulse oximetry, Gopikrishna et al. (2007b) [20] and Dastmalchi et al. (2012) [9] concluded that pulse oximetry is reliable, effective, and precise as a diagnostic tool for pulp vitality.

Other factors, such as ambient light, the transmitter light power, or surrounding tissues, have to be taken into consideration. The pulp is surrounded by hard tissues (enamel and dentine), which can have different thicknesses and morphologies, acting as an obstacle for the light, which scatters through the enamel prisms and dental tubules. The presence of restorative materials (i.e., composite resin, glass ionomer) and pulp cappers (i.e., calcium silicate cements) may also hinder the measurement since they can also act as an obstacle for the light source [36]. As a result, oxygen saturation levels shown in the pulse oximeter may be lower. Nevertheless, pulse oximetry could be investigated as a potential tool to monitor pulp vitality after vital pulp treatment or regenerative endodontic procedures $[37,38]$.

The pulp's anatomy and its distance from the sensor are other factors that could influence the reading. Moreover, age plays an important role, resulting in a series of time-dependent morphophysiological changes in the pulp complex-dentine continues its growth, and there could be deposition of mineralized tissue in the pulp and root canals [6]. This, combined with a decrease in pulpal blood flow, may hinder the dispersion of light, altering the pulse oximeter's reading. Regarding the influence of age on pulp vitality results using pulse oximetry, Stella et al. (2015) [33] and Estela et al. (2017b) [19] studied 
$\mathrm{SpO}_{2}$ levels among different age groups, concluding that younger participants had higher levels of saturation than those of greater age.

Regarding the quality assessment, the items regarding patient selection exhibited a low risk in all studies, although only one study enrolled a random sample [23]. Overall, 11 studies showed a high risk of bias concerning the index test $[4,9,11,15,17-19,23,27,31,32]$ since the results were interpreted while knowing the results of the reference standard, and another 11 studies showed high risk on the reference standard [11,21,22,24,26,28-33] because the results were interpreted knowing the results of the index test. In addition, all studies showed a low risk of bias considering flow and timing since all of them had an adequate interval between the index and reference standard tests and all patients received the same reference standard. Finally, all of the included studies presented a low risk of bias in the applicability concerns section since all of them matched their review questions and objectives.

The heterogeneity regarding the characteristics of the samples from the studies included in the present review (i.e., underlying pathologies, age, etc.) added to the differing methodologies used to assess pulse oximetry as a diagnostic tool for pulp vitality (i.e., pulse oximeter used, teeth assessed, etc.) made performing a quantitative analysis or meta-analysis of the results impracticable. For future studies in the field, the use of a standardized methodology is encouraged, in order to facilitate the quantitative analysis of the evidence as a whole.

The variety of results produced by the studies included in the present review may be influenced by a series of factors, namely, the range of sample sizes, teeth studied (which differ in size and shape), participant ages, pulse oximeter brands, and, generally, the methodology used. This acts as a limitation of the present systematic review. Additionally, the methodological heterogeneity of the included studies, added to the lack of published studies in the field and their variable quality, hinders the recommendation of a specific protocol for the clinical use of pulse oximetry as a diagnostic tool for dental pulp vitality in clinical practice. However, the qualitative synthesis performed may act as preliminary evidence of the potential application of pulse oximetry as an objective measurement of pulp vitality.

\section{Conclusions}

Although available evidence has demonstrated that pulse oximetry is a reliable and promising tool for the diagnosis of pulp vitality, it still has many limitations that hinder its use in daily dental practice. There is a need to design a specific pulse oximeter to use on teeth and a lack of studies that determine how $\mathrm{SpO}_{2}$ levels are affected in different circumstances such as systemic and oral pathologies, affecting the pulpar oxygenation.

Author Contributions: Conceptualization, L.F. and A.A.-G.; methodology, A.A.-G. and J.L.S.; software, J.L.S.; validation, L.F., C.L., M.M., and F.J.R.-L.; formal analysis, J.G.-G.; investigation, A.A.-G.; resources, M.M.; data curation, C.L.; writing—original draft preparation, A.A.-G.; writing-review and editing, J.L.S. and L.F.; visualization, J.G.-G. and M.M.; supervision, L.F., C.L., and F.J.R.-L.; project administration, M.M.; funding acquisition, M.M. All authors have read and agreed to the published version of the manuscript.

Funding: This research received no external funding.

Institutional Review Board Statement: Not applicable.

Informed Consent Statement: Not applicable.

Data Availability Statement: The data presented in this study are available on request from the corresponding author.

Conflicts of Interest: The authors declare no conflict of interest. 


\section{References}

1. Alghaithy, R.A.; Qualtrough, A. Pulp sensibility and vitality tests for diagnosing pulpal health in permanent teeth: A critical review. Int. Endod. J. 2017, 50, 135-142. [CrossRef]

2. Janani, K.; Palanivelu, A.; Sandhya, R. Diagnostic accuracy of dental oximeter with customized sensor holder, thermal test and electric pulp test for the evaluation of pulp vitality: An in vivo study. Braz. Dent. Sci. 2020, 23, 1-8. [CrossRef]

3. Ghouth, N.; Duggal, M.S.; BaniHani, A.; Nazzal, H. The diagnostic accuracy of laser Doppler flowmetry in assessing pulp blood flow in permanent teeth: A systematic review. Dent. Traumatol. 2018, 34, 311-319. [CrossRef] [PubMed]

4. Caldeira, C.L.; Barletta, F.B.; Ilha, M.C.; Abrão, C.V.; Gavini, G. Pulse oximetry: A useful test for evaluating pulp vitality in traumatized teeth. Dent. Traumatol. 2016, 32, 385-389. [CrossRef] [PubMed]

5. Chen, E.; Abbott, P.V. Dental pulp testing: A Review. Int. J. Dent. 2009. [CrossRef] [PubMed]

6. Pozzobon, M.H.; Sousa Vieira, R.; Hecke Alves, A.M.; Reyes-Carmona, J.; Silveira Teixeira, C.; Mendes de Souza, B.C.; Tadeu Felippe, W. Assessment of pulp blood flow in primary and permanent teeth using pulse oximetry. Dent. Traumatol. 2011, 27, 184-188. [CrossRef]

7. Jafarzadeh, H.; Rosenberg, P. Pulse Oximetry: Review of a potential aid in endodontic diagnosis. J. Endod. 2009, 35, 329-333. [CrossRef] [PubMed]

8. Bargrizan, M.; Ashari, M.A.; Ahmadi, M.; Ramezani, J. The use of pulse oximetry in evaluation of pulp vitality in immature permanent teeth. Dent. Traumatol. 2016, 32, 43-47. [CrossRef] [PubMed]

9. Dastmalchi, N.; Jafarzadeh, H.; Moradi, S. Comparison of the efficacy of a custom-made pulse oximeter probe with digital electric pulp tester, cold spray, and rubber cup for assessing pulp vitality. J. Endod. 2012, 38, 1182-1186. [CrossRef]

10. Gopikrishna, V.; Tinagupta, K.; Kandaswamy, D. Comparison of electrical, thermal and pulse oximetry methods for assessing pulp vitality in recently traumatized teeth. J. Endod. 2007, 35, 531-535. [CrossRef]

11. Kahan, R.S.; Gulabivala, K.; Snook, M.; Setchell, D.J. Evaluation of a pulse oximeter and customized probe for pulp vitality testing. J. Endod. 1996, 22, 105-109. [CrossRef]

12. Moher, D.; Liberati, A.; Tetzlaff, J.; Altman, D.G.; The PRISMA Group. Preferred reporting items for systematic reviews and meta-analyses: The PRISMA statement. PLoS Med. 2009, 6, e1000097. [CrossRef]

13. Liberati, A.; Altman, D.; Tetzlaff, J.; Mulrow, C.; Gøtzsche, P.; Loannidis, J.; Clarke, M.; Devereauz, J.; Kleijnen, J.; Moher, D. The PRISMA statement for reporting systematic reviews and meta-analyses of studies that evaluate health care interventions: Explanation and elaboration. J. Clin. Epidemiol. 2009, 62, e1-e34. [CrossRef]

14. Whiting, P.F.; Rutjes, A.W.S.; Westwood, M.E.; Mallett, S.; Deeks, J.J.; Reitsma, J.B.; Leeflang, M.M.G.; Sterne, J.A.C.; Bossuyt, P.M.M.; the QUADAS-2 Group. QUADAS-2: A revised tool for the quality assessment of diagnostic accuracy studies. Ann. Intern. Med. 2011, 155, 529-536. [CrossRef]

15. Anusha, B.; Madhusudhana, K.; Kumar Chinni, S.; Paramesh, Y. Assessment of pulp oxygen saturation levels by pulse oximetry for pulpal diseases-A diagnostic study. J. Clin. Diagn. Res. 2017, 11, 36-39. [CrossRef] [PubMed]

16. Bergesch, L.; Barletta, F.B.; Felippe, W.T.; Bruno, K.F.; Gonçalves de Alencar, A.H.; Estrela, C. Assessment of oxygen saturation in dental pulp of permanent teeth with periodontal disease. J. Endod. 2014, 40, 1927-1931.

17. Calil, E.; Caldeira, C.L.; Gavini, G.; Lemos, E.M. Determination of pulp vitality in vivo with pulse oximetry. Int. Endod. J. 2008, 41, 741-746. [CrossRef]

18. Estrela, C.; Oliveira, K.; Alencar, A.H.G.; Barletta, F.B.; Estrela, C.; Felippe, W.T. Oxygen saturation in the dental pulp of maxillary premolars in different age groups-Part 2. Braz. Dent. J. 2017, 28, 704-709. [CrossRef]

19. Estrela, C.; Serpa, G.C.; Alencar, A.H.; Bruno, K.F.; Barletta, F.B.; Felippe, W.T.; Estrela, C.; Souza, J.B. Oxygen saturation in the dental pulp of maxillary premolars in different age groups-Part 1. Braz. Dent. J. 2017, 28, 573-577. [CrossRef] [PubMed]

20. Gopikrishna, V.; Tinagupta, K.; Kandaswamy, D. Evaluation of efficacy of a new custom-made pulse oximeter dental probe in comparison with the electrical and thermal tests for assessing pulp vitality. J. Endod. 2007, 33, 411-414. [CrossRef]

21. Kataoka, S.H.; Setzer, F.C.; Gondim-Junior, E.; Faciola Pessoa, O.; Gavini, G.; Caldeira, C.L. Pulp vitality in patients with intraoral and oropharyngeal malignant tumors undergoing radiation therapy assessed by pulse oximetry. J. Endod. 2011, 36, 1197-1200. [CrossRef]

22. Kataoka, S.H.; Setzer, F.C.; Gondim-Junior, E.; Fregnani, E.R.; Moraes, C.J.; Faciola Pessoa, O.; Gavini, G.; Caldeira, C.L. Late effects of head and neck radiotherapy on pulp vitality assessed by pulse oximetry. J. Endod. 2016, 42, 886-889. [CrossRef] [PubMed]

23. Khademi, A.; Shahtouri, M.; Attar, B.; Rikhtergaran, N. Pulp vitality of maxillary canines after alveolar cleft bona grafting: Pulse oximetry versus electric pulp test versus cold test. J. Craniofacial Surg. 2017. [CrossRef]

24. Kong, H.J.; Jeon Shin, T.; Hyun, H.K.; Kim, Y.J.; Kim, J.K.; Shon, W.J. Oxygen saturation and perfusion index from pulse oximetry in adult volunteers with viable incisors. Acta Odontol. Scand. 2016, 74, 411-415. [CrossRef]

25. Schnettler, J.; Wallace, J. Pulse oximetry as a diagnostic tool of pulpal vitality. J. Endod. 1991, 17, 488-490. [CrossRef]

26. Setzer, F.; Kataoka, S.H.; Natrielli, F.; Gondim-Junior, E.; Caldeira, C. Clinical diagnosis of pulp inflammation based on pulp oxygenation rates measured by pulse oximetry. J. Endod. 2012, 38, 880-883. [CrossRef] [PubMed]

27. Solda, C.; Barletta, F.; Vanni, J.; Lambert, P.; Só, M.; Estrela, C. Effect of at-home bleaching on oxygen saturation levels in the dental pulp of maxilarry central incisors. Braz. Dent. J. 2018, 29, 541-546. [CrossRef] [PubMed] 
28. Souza, S.; Thomaz, E.; Costa, C. Healthy dental pulp oxygen saturation rates in subjects with homozygous sickle cell anemia: A cross-sectional study nested in a cohort. J. Endod. 2017, 43, 1997-2000. [CrossRef]

29. Karayilmaz, H.; Kirzioglu, Z. Comparison of the reliability of laser doppler flowmetry, pulse oximetry and electric pulp tester in assessing the pulp vitality of human teeth. J. Oral Rehabil. 2011, 38, 340-347. [CrossRef]

30. Kosturkov, D.; Uzunov, T. Pulse oximetry and electric pulp test in intact teeth and teeth with hyperaemia pulpae. Acta Med. Bulg. 2017, 44, 10-13. [CrossRef]

31. Kosturkov, D.; Uzunov, T.; Grozdanova, R.; Ivancheva, V. Evaluation of condition of the pulp by pulse oximetry. J. IMAB 2015, 21, 1003-1007. [CrossRef]

32. Sadique, M.; Ravi, S.V.; Thomas, K.; Dhanapal, P.; Simon, E.P.; Shaheen, M. Evaluation of efficacy of a pulse oximeter to assess pulp vitality. J. Int. Oral Health 2014, 6, 70-72. [PubMed]

33. Stella, J.P.; Branco Barletta, F.; Bergesch Giovanella, L.; Grazziotin-Soares, R.; Ferreira Tobo, M.; Tadeu Felippe, W.; Estrela, C. Oxygen saturation in dental pulp of permanent teeth: Difference between children/adolescents and adults. J. Endod. 2015, 41, 1445-1449. [CrossRef] [PubMed]

34. Levin, L. Pulp and periradicular testing. J. Endod. 2013, 39, 13-19. [CrossRef]

35. Mainkar, A.; Kim, S.G. Diagnostic accuracy of 5 dental pulp tests: A systematic review and meta-analysis. J. Endod. 2018, 44, 694-702. [CrossRef]

36. Bruno, K.; Brando Barletta, F.; Tadeau Felippe, W.; Almeida Silva, J.; Gonçalves de Alencar, A.H.; Estrela, C. Oxygen saturation in the dental pulp of permanent teeth: A critical review. J. Endod. 2014, 40, 1054-1057. [CrossRef]

37. Saoud, T.M.A.; Ricucci, D.; Lin, L.M.; Gaengler, P. Regeneration and Repair in Endodontics-A Special Issue of the Regenerative Endodontics-A New Era in Clinical Endodontics. Dent. J. 2016, 4, 3. [CrossRef]

38. Palma, P.J.; Martins, J.; Diogo, P.; Sequeira, D.; Ramos, J.C.; Diogenes, A.; Santos, J.M. Does Apical Papilla Survive and Develop in Apical Periodontitis Presence after Regenerative Endodontic Procedures? Appl. Sci. 2019, 9, 3942. [CrossRef] 